\title{
COVID19 EN POBLACIÓN RESIDENTE DE ZONAS GEOGRÁFICAS A ALTURAS SUPERIORES A 2500 m.s.n.m.
}

\author{
Cardona Rivero Anahí Karina1a \\ Montoya Lizárraga Manuel1b,2c \\ 1 Universidad Nacional de San Antonio Abad del Cusco. \\ 2 Hospital Regional del Cusco \\ a Docente Farmacia \\ b Docente Medicina Humana \\ c Especialista en infectología
}

\section{RESUMEN. -}

El objetivo del estudio fue evaluar las tasas de mortalidad por COVID19 en las regiones a alturas superiores de 2500 m.s.n.m. en comparación a las tasas de mortalidad por COVID19 de los países respectivos. Para lo cual se realizó un estudio de tipo descriptivo transversal comparativo, se revisaron 20 regiones de 7 países con ciudades de más de 100000 habitantes que se encuentran por encima de 2500 m.s.n.m. Se registraron los casos de fallecidos por COVID19, cantidad de población y las tasas de mortalidad al 30 de abril del 2020. Los resultados de las tasas de mortalidad fueron evaluados con pruebas de normalidad Kolmogorov y Smirnov y Shapiro Wilk. Para evaluar la diferencia entre las medias de las tasas de mortalidad se utilizó la prueba de Wilcoxon con signos para muestras relacionadas se trabajó a un nivel de confianza del 95\%, obteniendo un valor de $\mathrm{p}=$ $0,015<0,05$; que demuestra la diferencia estadísticamente significativa. Se concluye que el COVID19 presenta menores tasas de mortalidad en zonas geográficas con alturas superiores a 2500 m.s.n.m.

Palabras clave: altura, 2500 m.s.n.m., COVID19, tasa de mortalidad.

\section{ABSTRACT}

The objective of the study was to evaluate the mortality rates by COVID19 in the regions at altitudes higher than 2500 m.s.n.m., compared to the mortality rates by COVID19 of the respective countries. For this purpose, a comparative cross-sectional descriptive study was carried out, reviewing 20 regions of 7 countries with cities of more than 100,000 inhabitants that are above 2500 meters above sea level. Cases of deaths due to COVID19, population numbers and mortality rates as of April 30, 2020 were recorded. The results of the mortality rates were evaluated with Kolmogorov and Smirnov and Shapiro Wilk normality tests. To evaluate the difference between the means of mortality rates, the Wilcoxon test with signs for related samples was used at a 95\% confidence level, obtaining a value of $p=0.015<0.05$; which demonstrates the statistically significant difference. It is concluded that the COVID19 presents lower mortality rates in geographical areas with altitudes higher than 2500 meters above sea level

Keywords: altitude, 2500 m.s.n.m., COVID19, mortality rate 


\section{INTRODUCCIÓN. -}

Una emergencia en salud mundial actual es la pandemia del COVID-19, que se inició en China a fines del 2019 (1) y se está extendiendo a un ritmo exponencial, con millones de personas en todo el mundo en riesgo de contraer el síndrome respiratorio agudo severo (SARS-CoV-2) (2). Con alta mortalidad (3) y consecuencias no completamente conocidas (4).

También se reconoce que las complicaciones graves y consecuente muerte, dependen de múltiples factores (5), basados en la triada epidemiológica estos pueden ser factores del huésped, del patógeno y ambientales (6).

En cuanto a los factores ambientales la altura de las diferentes zonas geográficas puede influir en el curso de algunas patologías (7).

Por lo antes mencionado, el objetivo del presente estudio fue analizar la diferencia de las tasas de mortalidad de zonas geográficas a alturas superiores a 2500 msnm en relación las de mortalidad de sus respectivos países.

\section{MATERIALES Y MÉTODOS. -}

Se realizó un estudio descriptivo transversal comparativo de las tasas de mortalidad por COVID19 de las regiones y sus países respectivos. Para lo cual se usó la aplicación Google earth (8) para georreferenciar las regiones o departamentos. Los criterios de inclusión considerados son regiones de países con alturas superiores a $2500 \mathrm{msnm}$ en América, Asia y África, que tengan ciudades grandes con más de 100,000 habitantes para lo cual se revisaron los últimos censos de población, sin considerar otras medidas de contención y mitigación que se estén desarrollando para frenar la pandemia.

Se revisaron los casos y fallecidos por COVID19 para lo cual se utilizó el app the coronavirus (9) y la información de organismos de salud oficiales. Los datos se exportaron a una base Excel con la cual se calculó la tasa de mortalidad acumulada por 10000 habitantes al 30 de abril del 2020 de la región y del país correspondiente.

Para el análisis estadístico se utilizó el programa estadístico SPSS versión 18. Para la estadística bivariada se comparó la tasa de mortalidad de cada región con la tasa de mortalidad del país. Se aplicó la prueba de Kolmogorov y Smirnov y Shapiro Wilk para revisar la normalidad de las tasas de mortalidad, luego se usó la prueba no paramétrica de Wilcoxon prueba con signos para muestras relacionadas el cual se trabajó a un nivel de confianza del $95 \%$ y obteniendo un valor de $p=0,015<$ 0,05 ; que demuestra la diferencia estadísticamente significativa.

\section{RESULTADOS. -}

En la tabla 1, se observa la población total, el numero de fallecidos y las respectivas tasas de mortalidad al 30 de abril del 2020 de 7 países. En el caso de Perú se seleccionaron 6 regiones que cumplen con los criterios de inclusión, en Bolivia 5 regiones, en Ecuador 3, en Colombia 2 y México 2, en Chile y China 1. 
Tabla 1. Distribución de regiones con ciudades de más de 100,000 habitantes a una altura superior a los 2500 msnm según tasa de mortalidad por COVID 19

\begin{tabular}{|c|c|c|c|c|c|c|c|c|c|}
\hline CONTINENTE & PAÍS & Población ${ }^{1}$ & Fallecidos $^{2}$ & $\begin{array}{c}\text { Tasa de } \\
\text { mortalidad x } \\
10000 \text { hab. }\end{array}$ & $\begin{array}{c}\text { REGIÓN/ } \\
\text { DEPARTAMENTO }\end{array}$ & $\underset{3}{\operatorname{ALTITUD}}$ & $\begin{array}{c}\text { Población } \\
\text { por Región o } \\
\text { departament } \\
0^{4}\end{array}$ & $\begin{array}{l}\text { Falle } \\
\text { cidos } \\
2\end{array}$ & $\begin{array}{c}\text { Tasa de } \\
\text { mortalid } \\
\text { ad } x \\
10000 \\
\text { hab. }\end{array}$ \\
\hline \multirow[t]{17}{*}{$\begin{array}{l}\text { América del } \\
\text { Sur }\end{array}$} & \multirow{6}{*}{ Perú } & \multirow{6}{*}{33430992} & \multirow{6}{*}{1051} & \multirow{6}{*}{0.314} & Ancash & 3052 & 1083519 & 56 & 0.517 \\
\hline & & & & & Ayacucho & 2761 & 616176 & 0 & 0.000 \\
\hline & & & & & Cajamarca & 2750 & 1341012 & 3 & 0.022 \\
\hline & & & & & Cusco & 3399 & 1205527 & 3 & 0.025 \\
\hline & & & & & Junín & 4107 & 1246038 & 4 & 0.032 \\
\hline & & & & & Puno & 3810 & 1172697 & 0 & 0.000 \\
\hline & \multirow{5}{*}{ Bolivia } & \multirow{5}{*}{11560300} & \multirow{5}{*}{59} & \multirow{5}{*}{0.051} & Chuquisaca & 2554 & 581347 & 0 & 0.000 \\
\hline & & & & & Cochabamba & 2574 & 1930143 & 4 & 0.021 \\
\hline & & & & & La Paz & 3640 & 2719344 & 11 & 0.040 \\
\hline & & & & & Oruro & 3729 & 494587 & 2 & 0.040 \\
\hline & & & & & Potosí & 3977 & 823517 & 1 & 0.012 \\
\hline & \multirow{3}{*}{ Ecuador } & \multirow{3}{*}{17398999} & \multirow{3}{*}{900} & \multirow{3}{*}{0.517} & Chimborazo & 3900 & 502004 & 37 & 0.737 \\
\hline & & & & & Pichincha & 2953 & 3228233 & 75 & 0.232 \\
\hline & & & & & Tungurahua & 2500 & 590600 & 19 & 0.322 \\
\hline & \multirow{2}{*}{ Colombia } & \multirow{2}{*}{50575099} & \multirow{2}{*}{293} & \multirow{2}{*}{0.058} & Boyacá & 3427 & 1242731 & 10 & 0.080 \\
\hline & & & & & Cundinamarca & 3341 & 3242999 & 10 & 0.031 \\
\hline & Chile & 18894941 & 227 & 0.120 & \begin{tabular}{|l|} 
Arica y \\
Parinacota \\
\end{tabular} & 3753 & 226068 & 4 & 0.177 \\
\hline Asia & China & 1410929134 & 4633 & 0.033 & Tibet & 4900 & 3180000 & 1 & 0.003 \\
\hline \multirow[t]{3}{*}{$\begin{array}{l}\text { América del } \\
\text { Norte }\end{array}$} & \multirow{2}{*}{ México } & \multirow{2}{*}{135684001} & \multirow{2}{*}{1930} & \multirow{2}{*}{0.142} & Chiapas & 4080 & 5217908 & 6 & 0.011 \\
\hline & & & & & Estado México & 2605 & 16187608 & 159 & 0.098 \\
\hline & TOTAL & & & & 20 & & & & \\
\hline
\end{tabular}

En la tabla 2 se observa las pruebas de normalidad. La prueba de Kolmogorov Smirnov se obtiene para el G1 p=0,016 <0,05 y G2 p=0,000 < 0,05 y para la prueba de Shapiro -Wilk G1 $p=0,002<0,05$ y $G 2 p=0,000<0,05$. En base a los resultados obtenidos los datos no tienen distribución normal. En consecuencias para evaluar la variación de las tasas de mortalidad se aplicó la prueba de Wilcoxon de los rangos con signos para muestras relacionadas, obteniendo un valor $p=0,015<0,05$; que demostraría la diferencia estadísticamente significativa. 
Tabla Na 2. Pruebas estadísticas de normalidad y comparación de medias para muestra relacionadas

\begin{tabular}{|c|c|c|c|c|c|c|c|}
\hline & \multicolumn{3}{|c|}{ Kolmogorov-Smirnov } & \multicolumn{3}{|c|}{ Shapiro-Wilk } & \multirow{2}{*}{$\begin{array}{c}\text { Prueba de Wilcoxon } \\
\text { Sig. }\end{array}$} \\
\hline & Estadístico & $\mathrm{gl}$ & Sig. & Estadístico & gl & Sig. & \\
\hline $\begin{array}{l}\text { Tasa mortalidad } \\
\text { país (G1) }\end{array}$ & 0,215 & 20 & 0,016 & 0,827 & 20 & 0,002 & \\
\hline $\begin{array}{l}\text { Tasas mortalidad } \\
\text { región o } \\
\text { departamento } \\
\text { (G2) }\end{array}$ & 0,308 & 20 & 0,000 & 0,653 & 20 & 0,000 & 0.015 \\
\hline
\end{tabular}

\section{DISCUSIÓN}

La diferencia significativa encontrada en la tabla 2 sugiere que las tasas de mortalidad en las regiones con altitud mayor a $2500 \mathrm{msnm}$ en relación la tasa de mortalidad global del país. La variación de las tasas de mortalidad del G1 (país) en relación a (G2) (regiones), evidencian una diferencia significativa el valor $p=0.015<0,05$ obtenido mediante la prueba no paramétrica de Wilcoxon de los rangos con signo para muestras relacionadas.

Con más de 100 días de declarada pandemia (15), que afecta a más de 120 países (16), se puede percibir que la incidencia de casos es diferente en las regiones dentro de un país relacionada con la altura. En los primeros días de la pandemia en muchas regiones los casos no fueron detectados oportunamente por falta de pruebas diagnósticas lo cual significó un sub registro lo que pudo sesgar las estadísticas, después de más de 3 meses desde el inicio de los primeros casos, se observa que la diferencia es significativa en los casos de COVID19 a nivel en ciudades grandes (más de 100000 habitantes) a más de 2500 msnm frente a la tasa de sus países respectivos. Los informes iniciales sugieren que la hipertensión, la diabetes y las enfermedades cardiovasculares fueron las comorbilidades más frecuentes en los pacientes afectados, y las tasas de letalidad tienden a ser altas en individuos afectados por el COVID-19 (17). En la evaluación podemos evidenciar que las complicaciones con subsecuente fallecimiento no son elevadas en regiones con alturas superiores a $2500 \mathrm{msnm}$.

El presente estudio coincide con los hallazgos de Arias y col. (18), que evaluaron las regiones de mayor altura el Tibet en China, Bolivia y Ecuador donde se observó correlación entre la mayor altura y menos casos positivos de COVID19.

Cerca de 140 millones de personas en el mundo viven a alturas mayores a los 2500 msnm (19), siendo este el punto de corte que se considera a poblaciones sujetas a hipoxia hipobárica por lo que son pobladores que se han adaptado durante milenios patrones fenotípicos que los protegen de efectos deletéreos por la hipoxia crónica (20). Una condición del habitante de altura son sus características genéticas y su relación con el Sistema Renina Angiotensina con menor presencia de receptores ECA2 en las células principalmente en el sistema respiratorio (21). Si esta condición se correlaciona con el comportamiento del virus, se observa que el agente tiene tropismo por los receptores ECA2, uniéndose el virus mediante su proteína $S$ viral, accediendo a las células del huésped para cumplir su ciclo de replicación (22) 
Es importante considerar en nuestro análisis que los pacientes repartidos en ciudades capitales de países como Quito (Pichincha Ecuador) con 800 casos y 40 decesos por COVID19 tienen más de dos millones de habitantes, lo mismo sucede con Bogotá (Cundinamarca Colombia) con 1883 casos y 73 decesos por COVID19 con más de siete millones de habitantes. Los establecimientos de salud de estas ciudades son sitios de referencia para pacientes críticos pueden incluir pacientes importados graves dentro de sus cifras de casos.

En muchos países el impacto de la gravedad del COVID19, se enfoca en áreas que tienen mayor cantidad de casos, lo cual es lógico. Cuando se realizan estimaciones más precisas sobre la localización, identificación y seguimiento de casos son variables de un país a otro y están influenciados por la fase de la epidemia que puede ser de contención o de diseminación comunitaria. Por lo que recaudar la información de casos es difícil con exactitud. Como sucedió en el presente estudio donde se tuvo que contrastar cifras nacionales oficiales y la información local.

Fuentes de financiamiento: autofinanciado.

Conflictos de interés: la autora declara que no hay ningún conflicto de interés en la publicación del presente artículo.

\section{REFERENCIAS BIBLIOGRÁFICAS}

1. Chen N, Zhou M, Dong X, Qu J, Gong F, Han Y, et al. Epidemiological and clinical characteristics of 99 cases of 2019 novel coronavirus pneumonia in Wuhan, China: a descriptive study. Lancet [Internet]. 2020 Feb 15 [cited 2020 Apr 16];395(10223):507-13. Available from: https://www.sciencedirect.com/science/article/pii/S0140673620302117

2. Ghosal S, Sengupta S, Majumder M, Sinha B. Linear Regression Analysis to predict the number of deaths in India due to SARS-CoV-2 at 6 weeks from day 0 (100 cases - March 14th 2020). Diabetes Metab Syndr Clin Res Rev [Internet]. 2020 Jul 1 [cited 2020 Apr 16];14(4):311-5. Available from: https://www.sciencedirect.com/science/article/pii/S1871402120300576

3. Sohrabi C, Alsafi Z, O'Neill N, Khan M, Kerwan A, Al-Jabir A, et al. World Health Organization declares global emergency: A review of the 2019 novel coronavirus (COVID-19). Int J Surg [Internet]. 2020 Apr 1 [cited 2020 Apr 16];76:71-6. Available from: https://www.sciencedirect.com/science/article/pii/S1743919120301977

4. Troyer EA, Kohn JN, Hong S. Are we facing a crashing wave of neuropsychiatric sequelae of COVID-19? Neuropsychiatric symptoms and potential immunologic mechanisms. Brain Behav Immun [Internet]. 2020 Apr 13 [cited 2020 Apr 16]; Available from: https://www.sciencedirect.com/science/article/pii/S088915912030489X

5. Zhou F, Yu T, Du R, Fan G, Liu Y, Liu Z, et al. Clinical course and risk factors for mortality of adult inpatients with COVID-19 in Wuhan, China: a retrospective cohort study. Lancet [Internet]. 2020 Mar 28 [cited 2020 Apr 16];395(10229):1054-62. Available from: https://www.sciencedirect.com/science/article/pii/S0140673620305663

6. Jones KE, Patel NG, Levy MA, Storeygard A, Balk D, Gittleman JL, et al. Global trends in emerging infectious diseases. Nature. 2008;451(7181):990-3.

7. Shouval DS, Rufo PA. The Role of Environmental Factors in the Pathogenesis of 
Inflammatory Bowel Diseases: A Review. JAMA Pediatr [Internet]. 2017 Oct 1;171(10):9991005. Available from: https://doi.org/10.1001/jamapediatrics.2017.2571

8. GoogleEarth [Internet]. [cited 2020 Apr 26]. Available from: https://earth.google.com/web/@1.18327072,-160.42678609,27576.55708151a,41450411.0659361d,35y,0h,0t,0r

9. The coronavirus app [Internet]. [cited 2020 Apr 30]. Available from: https://coronavirus.app/tracking/

10. Countrymeters [Internet]. [cited 2020 Apr 30]. Available from: https://countrymeters.info/es/

11. Instituto Naciona de Salud y Ministerio de Salud Peru. Sala Situacional COVID 19 Perú [Internet]. Instituto Naciona de Salud y Ministerio de Salud Peru. 2020. Available from: https://community.powerbi.com/t5/Data-Stories-Gallery/Perú-Sala-Situacional-COVID-19Perú-Covid-19-Report/td-p/1019001

12. MINSALUD (Ministerio de Salud de Colombia). Coronavirus COVID19 [Internet]. Ministerio de Salud Colombia. 2020. Available from: https://d2jsqrio60m94k.cloudfront.net/

13. Balash E, Judeikyte E. Ministerio de Salud Pública de Ecuador) Comportamiento COVID19 Ecuador [Internet]. 2020 [cited 2020 Apr 30]. Available from: https://public.tableau.com/profile/publicaciones.dneais.msp\#!/vizhome/COVID19ecu_MSP _15866333883550/COVID-19MSP

14. MINSAL Chile (Ministerio de Salud de Chile). Plan de Acción Coronavirus COVID 19 [Internet]. Ministerio de Salud Chile. 2020 [cited 2020 Apr 30]. Available from: https://www.minsal.cl/nuevo-coronavirus-2019-ncov/casos-confirmados-en-chile-covid19/

15. Eurosurveillance Editorial Team. Note from the editors: World Health Organization declares novel coronavirus (2019-nCoV) sixth public health emergency of international concern. Euro Surveill. 2020;25(5):2019-20.

16. WHO. Coronavirus Disease 2019. A \& A Practice. 2020;e01218.

17. Belén M, De La Cruz J, Garcia L, Santiango A. Influencia de la altitud sobre la regulaciónde la presión arterial en el ejercicio físico efectos sobre el sistema renina-angiotensinaaldosterona Archivos de medicina del deporte. Arch Med del Deport [Internet]. 2004;21:287-2965. Available from: http://archivosdemedicinadeldeporte.com/articulos/upload/Original_influencia_287_102. pdf

18. Arias-Reyes C, Zubieta-DeUrioste N, Poma-Machicao L, Aliaga-Raduan F, Carvajal-Rodriguez $\mathrm{F}$, Dutschmann $\mathrm{M}$, et al. Does the pathogenesis of SARS-CoV-2 virus decrease at highaltitude? Respir Physiol Neurobiol [Internet]. 2020 Jun;277:103443. Available from: https://linkinghub.elsevier.com/retrieve/pii/S1569904820301014

19. Moore LG, Niermeyer S, Zamudio S. Human adaptation to high altitude: Regional and lifecycle perspectives. Am J Phys Anthropol. 1998;107(S27):25-64.

20. Bigham AW. Genetics of human origin and evolution: high-altitude adaptations. Curr Opin Genet Dev [Internet]. 2016;41:8-13. Available from:

http://dx.doi.org/10.1016/j.gde.2016.06.018

21. Cheng $\mathrm{H}$, Wang $\mathrm{Y}$, Wang $\mathrm{G}-\mathrm{Q}$. Organ-protective effect of angiotensin-converting enzyme 2 
and its effect on the prognosis of COVID-19. J Med Virol. 2020;(March):1-5.

22. Hoffmann $M$, Kleine-Weber H, Krüger N, Müller M, Drosten C, Pöhlmann S. The novel coronavirus 2019 (2019-nCoV) uses the SARS-coronavirus receptor ACE2 and the cellular protease TMPRSS2 for entry into target cells. bioRxiv [Internet]. 2020 Jan 1;2020.01.31.929042. Available from: http://biorxiv.org/content/early/2020/01/31/2020.01.31.929042.abstract 